\title{
N131: A dust bubble born from the disruption of a gas filament ${ }^{\star}$
}

\author{
Chuan-Peng Zhang ${ }^{1,2,5,6}$, Guang-Xing $\mathrm{Li}^{2,3}$, Friedrich Wyrowski ${ }^{2}$, Jun-Jie Wang ${ }^{1,5}$, Jing-Hua Yuan ${ }^{1}$, Jin-Long Xu ${ }^{1,5}$, \\ Yan Gong ${ }^{2,4,6}$, Cosmos C. Yeh ${ }^{2}$, and Karl M. Menten ${ }^{2}$ \\ ${ }^{1}$ National Astronomical Observatories, Chinese Academy of Sciences, Beijing, 100012, PR China \\ e-mail: cpzhang@nao.cas.cn \\ 2 Max-Planck-Institut für Radioastronomie, Auf dem Hügel 69, 53121 Bonn, Germany \\ 3 University Observatory Munich, Scheinerstrasse 1, 81679 Munich, Germany \\ ${ }^{4}$ Purple Mountain Observatory \& Key Laboratory for Radio Astronomy, Chinese Academy of Sciences, 2 West Beijing Road, \\ Nanjing, 210008, PR China \\ 5 NAOC-TU Joint Center for Astrophysics, Lhasa, 850000, PR China \\ ${ }^{6}$ University of the Chinese Academy of Sciences, Beijing, 100080, PR China
}

Received 10 April 2015 / Accepted 16 October 2015

\section{ABSTRACT}

Context. OB-type stars have strong ionizing radiation and drive energetic winds. The ultraviolet radiation from ionizing stars may heat dust and ionize gas to sweep up an expanding bubble shell. This shell may be the result of feedback leading to a new generation of stars.

Aims. N131 is an infrared dust bubble residing in a molecular filament. We study the formation and fragmentation of this bubble with multiwavelength dust and gas observations.

Methods. Towards the bubble N131, we analysed archival multiwavelength observations including 3.6, 4.5, 5.8, 8.0, 24, 70, 160, 250, $350,500 \mu \mathrm{m}, 1.1 \mathrm{~mm}$, and $21 \mathrm{~cm}$. In addition, we performed new observations of $\mathrm{CO}(2-1), \mathrm{CO}(1-0)$, and ${ }^{13} \mathrm{CO}(1-0)$ with the IRAM $30 \mathrm{~m}$ telescope.

Results. Multiwavelength dust and gas observations reveal a ring-like shell with compact fragments, two filamentary structures, and the secondary bubble N131-A. Bubble N131 is a rare object with a large hole at $24 \mu \mathrm{m}$ and $21 \mathrm{~cm}$ in the direction of its centre. The dust and gas clumps are compact and might have been compressed at the inner edge of the ring-like shell, while they are extended and might be pre-existing at the outer edge. The column density, excitation temperature, and velocity show a potentially hierarchical distribution from the inner to outer edge of the ring-like shell. We also detected the front and back sides of the secondary bubble N131$\mathrm{A}$ in the direction of its centre. The derived Lyman-continuum ionizing photon flux within N131-A is equivalent to an O9.5 star. Based on the above, we suggest that the bubble N131 might be triggered by the strong stellar winds from a group of massive stars inside the bubble.

Conclusions. We propose a scenario in which the bubble N131 forms from the disruption of a gas filament by the expansion of the H II region, strong stellar winds, and fragments under self-gravity.

Key words. infrared: ISM - stars: formation - ISM: bubbles - ISM: clouds

\section{Introduction}

Interstellar bubbles are very common objects, but their expansion is an important mechanism that reshapes the morphology of the interstellar medium (Churchwell et al. 2006, 2007; Simpson et al. 2012; Hou \& Gao 2014). The formation of bubbles is still unclear (e.g. Beaumont \& Williams 2010). Generally, most of the bubbles exhibit an expanding $\mathrm{H}$ II region enclosed within the bubble (Deharveng et al. 2010). It is likely that bubbles are three-dimensional (3D) structures with a blueshifted front side and a redshifted back side, but the front and back sides are difficult to detect (e.g. Peng et al. 2010; Zhang \& Wang 2012; Beaumont \& Williams 2010). If a bubble is expanding, we should easily observe the expansion of its ring-like shell; however, just a few examples show the signatures of expanding shells, i.e. N6 (Yuan et al. 2014), N68 (Zhang \& Wang 2013), and N131 (Zhang et al. 2013). Frequently, we observe pillars and shell-like structures from dust emission along the expansion

\footnotetext{
* The reduced datacubes (FITS files) are only available at the CDS via anonymous ftp to cdsarc.u-strasbg. fr (130.79.128.5) or via http://cdsarc.u-strasbg.fr/viz-bin/qcat?J/A+A/585/A117
}

direction (e.g. the bubble CN88 in Churchwell et al. 2007), which might provide clues to the origin of bubbles. Ring-like shells may be triggered through the "collect-and-collapse" process (Whitworth et al. 1994b), the radiation driven implosion of pre-existing dense molecular clumps (Lefloch \& Lazareff 1994; Deharveng et al. 2010), stellar winds (Dyson \& Williams 1980), and supernova explosions (Churchwell et al. 2006), which take part in shaping the morphological structure of the bubble, such as pillars and shell-like structures. Young stellar objects (YSOs) and IRAS sources are often distributed among ring-like shells. These newly formed stars are likely triggered by the expansion of bubbles (Castor et al. 1975; Weaver et al. 1977; Watson et al. 2008; Zhang \& Wang 2012; Cappa et al. 2014).

Is there any connection between the formations of bubbles and filaments? Filamentary structures in molecular clouds are ubiquitous in the Milky Way, and have been identified from subparsec to kpc scale (Wang et al. 2011; Jackson et al. 2010; Li et al. 2013; Peretto et al. 2014; Ragan et al. 2014; Goodman et al. 2014). On the molecular cloud scale, filaments may form through converging flows (Csengeri et al. 2011), shear (Smith et al. 2014), the collision of shocked sheets (Padoan et al. 2001), 


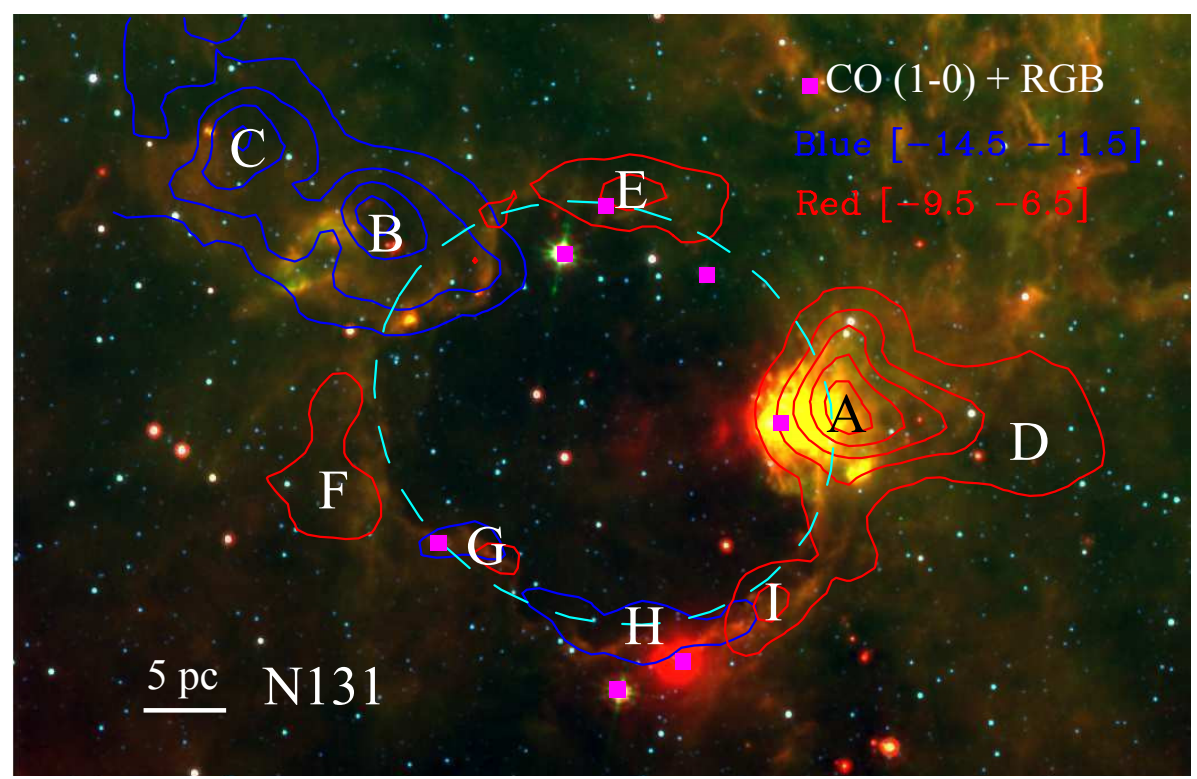

Fig. 1. Integrated intensity contours of the $\mathrm{CO}(1-0)$ line of the blueshifted and redshifted clouds superimposed on the RGB image with $24 \mu \mathrm{m}$ (red), $8.0 \mu \mathrm{m}$ (green), and $4.5 \mu \mathrm{m}$ (blue). The $\mathrm{CO}(1-0)$ data is from Zhang et al. (2013). The integration range is from -14.5 to $-11.5 \mathrm{~km} \mathrm{~s}{ }^{-1}$ for the blueshifted cloud, and from -9.5 to $-6.5 \mathrm{~km} \mathrm{~s}^{-1}$ for the redshifted cloud. The symbols "a", letters (A, B, ..., and I), and ellipse indicate the positions of eight IRAS point sources, nine molecular clumps, and ring-like shell of the bubble, respectively.

instabilities in self-gravitating sheets (Nagai et al. 1998), or turbulence. They can evolve to form stars (Kainulainen et al. 2013; Gómez et al. 2011; Wang et al. 2014), and in the final stage the star formation process should back-react on the filamentary structures. This feedback can either trigger the formation of the next generation of stars (Whitworth et al. 1994a) or disrupt the whole structure. However this feedback has not been studied in detail. If a cluster of massive stars resides within a filament, the strong stellar winds from these stars may disrupt the filament's structure. Such a scenario might be ongoing in the dust bubble N131 and its associated molecular clouds.

A promising object that might provide new insight into the formation of bubbles and their relation to filaments is the infrared dust bubble N131. Based on previous work from Zhang et al. (2013), we present its composite colour image in Fig. 1. This bubble was selected from the catalogue published by Churchwell et al. (2006). Its intriguing molecular structure was first observed and investigated with three $\mathrm{CO}(1-0)$ isotopic variants by Zhang et al. (2013). Bubble N131 has an inner minor radius of $13.0 \mathrm{pc}$ and inner major radius of $15.0 \mathrm{pc}$ at a kinematic distance of $8.6 \mathrm{kpc}$ with a centre coordinate of $\operatorname{RA}(\mathrm{J} 2000)=19^{\mathrm{h}} 52^{\mathrm{m}} 21^{\mathrm{s}} .5$, Dec. $(\mathrm{J} 2000)=+26^{\circ} 21^{\prime} 24^{\prime \prime} .0$ (Zhang et al. 2013). The $\mathrm{CO}$ emission is well correlated with a ringlike shell of Spitzer 24 and $8.0 \mu \mathrm{m}$ emission. There are two giant elongated molecular clumps (named clumps AD and BC) appearing on opposite sides of the ring-like shell of N131 (see Fig. 1). Clump BC at the north-east location has a velocity range of $[-14.5-11.5] \mathrm{km} \mathrm{s}^{-1}$, while clump AD at the south-west location is in the range of $[-9.5-6.5] \mathrm{km} \mathrm{s}^{-1}$. In addition, there is a huge cavity inside the bubble seen in the $24 \mu \mathrm{m}$ and $1.4 \mathrm{GHz}$ emission, which suggests that the ionized gas and hot dust may be evacuated by strong stellar winds. There are seven IRAS point sources located along the ring-like shell and well correlated with several molecular clumps. By revealing the distributions of YSOs, Zhang et al. (2013) found that there are 15 ionizing stars located within the bubble, and 63 YSOs surrounding the bubble. These may be the result of an expansion of strong stellar winds within the bubble.
The dynamics of bubble N131 is very intriguing, especially the two giant molecular filaments with blueshifted and redshifted velocity components (Zhang et al. 2013). However, the origin of its dynamical properties is not known. Investigating physical parameters such as the density, temperature, and velocity will help us to understand the formation of bubbles because these parameters may show a hierarchical condition of the associated clumps. By studying the morphology we might be able to understand its past. Bubble N131 is an excellent object for carrying out such a project. In this work, we investigate the formation and evolution of N131, using higher angular resolution CO observations, along with archival $3.6 \mu \mathrm{m}$ to $21 \mathrm{~cm}$ continuum data. In Sect. 2 we describe the observations and data reduction. In Sect. 3 we analyse the observational results from different wavelengths for the dust and gas clumps. In Sect. 4 we study the fragmentation and subsequent star formation of the bubble N131. In Sect. 5 the results are summarized.

\section{Observations and data}

\subsection{CO observations}

We simultaneously carried out $\mathrm{CO}(2-1), \mathrm{CO}(1-0)$, and ${ }^{13} \mathrm{CO}$ (1-0) observations during 2014 April 17-20 using the IRAM $30 \mathrm{~m}$ telescope ${ }^{1}$ on Pico Veleta, Spain. The E90 and E230 bands of the new Eight MIxer Receiver (EMIR) covered the three lines. The receiver was tuned to cover the frequency ranges 110.186 to $112.006 \mathrm{GHz}$ for ${ }^{13} \mathrm{CO}(1-0), 113.466$ to $115.286 \mathrm{GHz}$ for $\mathrm{CO}(1-0)$, and 229.678 to $231.498 \mathrm{GHz}$ for CO (2-1) observations. The Fast Fourier Transform Spectrometer (FFTS) backends were set at $50 \mathrm{kHz}$ resolution.

For the CO (2-1), CO (1-0), and ${ }^{13} \mathrm{CO}(1-0)$ lines, the half-power beam width (HPBW) was 11.3", 22.5", and 23.5", the main beam efficiency $\left(B_{\text {eff }}\right)$ was $59 \%, 78 \%$, and $78 \%$, and the forward efficiency $\left(F_{\text {eff }}\right)$ of the IRAM 30 m telescope was

1 Based on observations carried out with the IRAM 30 m Telescope. IRAM is supported by INSU/CNRS (France), MPG (Germany) and IGN (Spain). 
$92 \%, 94 \%$, and $94 \%$, respectively. The relation between main beam temperature $\left(T_{\mathrm{mb}}\right)$ and antenna temperature $\left(T_{\mathrm{A}}^{*}\right)$ is $T_{\mathrm{mb}}=$ $\left(F_{\text {eff }} / B_{\text {eff }}\right) \times T_{\mathrm{A}}^{*}$. The on-the-fly mapping mode was used to scan the bubble shell in two orthogonal directions, to reduce striping on the maps. Given limited observing time, the sampling step was set as 9.3", which meets the Nyquist sampling theorem for $\mathrm{CO}(1-0)$ and ${ }^{13} \mathrm{CO}(1-0)$, yet not for CO $(2-1)$. However, this was ameliorated by scanning in the two orthogonal directions.

Calibration scans, pointing, and focus were done on a regular basis to assure correct calibration. Calibration scans were done at the beginning of each subscan. A pointing was done about every hour, and a focus scan every three hours with more scans performed around sunset and sunrise. The flux calibration is expected to be accurate within $10 \%$. The GILDAS ${ }^{2}$ and MIRIAD ${ }^{3}$ software packages were used to reduce the observational data.

\subsection{Archival data}

In Fig. 2, multiwavelength observations are shown for bubble N131 from $3.6 \mu \mathrm{m}$ to $21 \mathrm{~cm}$. The combined data comprise GLIMPSE 3.6, 4.5, 5.8, $8.0 \mu \mathrm{m}$ (Benjamin et al. 2003; Churchwell et al. 2009); MIPSGAL $24 \mu \mathrm{m}$ (Carey et al. 2009); PACS 70 and $160 \mu \mathrm{m}$ (Poglitsch et al. 2010); SPIRE 250, 350, and $500 \mu \mathrm{m}$ (Griffin et al. 2010); BGPS $1.1 \mathrm{~mm}$ (Aguirre et al. 2011; Ginsburg et al. 2013); and NVSS $21 \mathrm{~cm}$ (Condon et al. 1998). These data were direct archive products. The brightest region (or saturated region) with dashed square was zoomed in, and then shown in the bottom left corner of each subfigure as a zoom-in with adjusted intensity scale.

\section{Analysis and results}

\subsection{Multiwavelength dust observations}

The 3.6 and $4.5 \mu \mathrm{m}$ emission mostly originates from some stars associated with N131 or bright field stars. The emission at both wavelengths does not delineate the shell structure of the bubble, except for several bright stars in Fig. 2. These bright stars are associated with IRAS sources (Zhang et al. 2013). Zoomed in the dashed square, the 3.6 and $4.5 \mu \mathrm{m}$ emission shows a cometary morphology (named N131-A) with a wide angle tail. The 5.8 and $8.0 \mu \mathrm{m}$ emission originates mainly from polycyclic aromatic hydrocarbons (PAHs; Watson et al. 2008), which are excited by the photodissociation region (PDR) at the interface between the wind-blown bubble and the ambient interstellar medium. The shell structure begins to be visible at $5.8 \mu \mathrm{m}$, and becomes prominent at $8.0 \mu \mathrm{m}$.

The 24 and $70 \mu \mathrm{m}$ emission is mostly produced by relatively hot dust. The $70 \mu \mathrm{m}$ emission corresponds to hot dust emission of $\geqslant 40 \mathrm{~K}$ (Faimali et al. 2012), and partly to cool dust emission (Anderson et al. 2012). Deharveng et al. (2010) found that about $98 \%$ of the bubbles exhibit extended and prominent $24 \mu \mathrm{m}$ emission enclosed within the bubble. In Fig. 2, however, the $24 \mu \mathrm{m}$ emission clearly delineates the ring-like shell of bubble $\mathrm{N} 131$. Inside the bubble only a weak patch of $24 \mu \mathrm{m}$ emission is found. It is associated with an unrelated velocity component at $25.0 \mathrm{~km} \mathrm{~s}^{-1}$ (Zhang et al. 2013). So, the bubble shows a large cavity at $24 \mu \mathrm{m}$ inside the bubble. The inner edge of the shell is brighter than the outer edge at $24 \mu \mathrm{m}$, which shows an apparent intensity gradient (see Sect. 3.5). Along the ring-like shell at $24 \mu \mathrm{m}$, there are also several bright point sources associated

\footnotetext{
2 http://www.iram.fr/IRAMFR/GILDAS/

3 http://www.cfa.harvard.edu/sma/miriad/
}

with IRAS sources (Zhang et al. 2013). This is likely from feedback of the shocked stellar winds within the bubble. For N131-A region (see Fig. 2) rescaled within the dashed square, the morphology of the $24 \mu \mathrm{m}$, showing a bright and compact spot, is not similar to 3.6-8.0 $\mu \mathrm{m}$ emission. We suggest that N131-A is actually a secondary bubble (see Sect. 3.7). At $70 \mu \mathrm{m}$, the emission from the ring-like shell becomes weak, yet still visible. N131-A in the zoomed square becomes very bright with extended tails at $70 \mu \mathrm{m}$.

The 160, 250, 350, and $500 \mu \mathrm{m}$ emission originates from cool dust with an average temperature of $26 \mathrm{~K}$ along the PDR (Anderson et al. 2012). This cool dust may be from the extended envelope of the ring-like shell. From 160 to $500 \mu \mathrm{m}$, the dust structure becomes more extended, partly because of the lower spatial resolution and because the longer wavelengths trace colder and more diffuse regions than the PDR shell. Next to the west side of the bubble there is a large ridge extending from north to south. This ridge has no detected velocity component associated with the bubble, so it may be partly from an unrelated background or foreground. Inside the bubble, one dust clump is visible. This clump is associated with another CO velocity component at $25.0 \mathrm{~km} \mathrm{~s}^{-1}$ (Zhang et al. 2013), excluding the possibility of it being the front side and back side of a 3D bubble structure (e.g. Zhang \& Wang 2012). Within the zoomed square, N131-A has two visible tails. Outside the square, N131A also has two filamentary clumps towards the west. We propose that it is due to feedback of the stellar winds inside the bubble.

The $1.1 \mathrm{~mm}$ emission is dominated by cold dust. At limited sensitivity, we can just see an extended clump associated with N131-A in Fig. 2. The $21 \mathrm{~cm}$ emission is mainly from freefree emission, which traces $\mathrm{H}$ II regions. The $21 \mathrm{~cm}$ emission is also coincident with N131-A within the square. The peak of the $21 \mathrm{~cm}$ emission is located at the peak of the secondary bright point source (see Sect. 3.7).

\subsection{Fragment extraction}

To study the fragmentation in the ring-like shell of the bubble N131, the Gaussclumps algorithm in GILDAS was used to extract dense fragments (Stutzki \& Guesten 1990; Kramer et al. 1998). Gaussclumps identifies local maxima in images and fits them with Gaussian intensity distributions. When fitting a Gaussian to the maximum, a modified chi-squared function is minimized. Three "stiffness" parameters control the fitting, ensuring that a local clump is fitted and subtracted. We only considered those fragments with the peaks of column density above $5 \sigma$ and with the fitted Gaussian FWHMs larger than their beam size. The derived parameters are listed in Table 1, namely position, size, column density, and mass. The column densities and masses are calculated in Sect. 3.9.

\subsection{Morphology of molecular clumps}

Earlier, lower-resolution ${ }^{12} \mathrm{CO}(1-0)$ observations have identified nine extended molecular clumps surrounding the shell of N131 (labelled A-I; see Fig. 2 of Zhang et al. 2013). The nine clumps were found surrounding a cavity which opens towards the north. An apparent intensity gradient from the inner to the outer edge of the ring-like shell was revealed in addition to two giant elongated molecular clumps at the opposite sides of the ring-like shell of N131. For the two molecular clumps, the brightest positions in ${ }^{12} \mathrm{CO}(1-0)$ distribution are located at the ring-like shell, and then the emission gradually becomes diffuse 

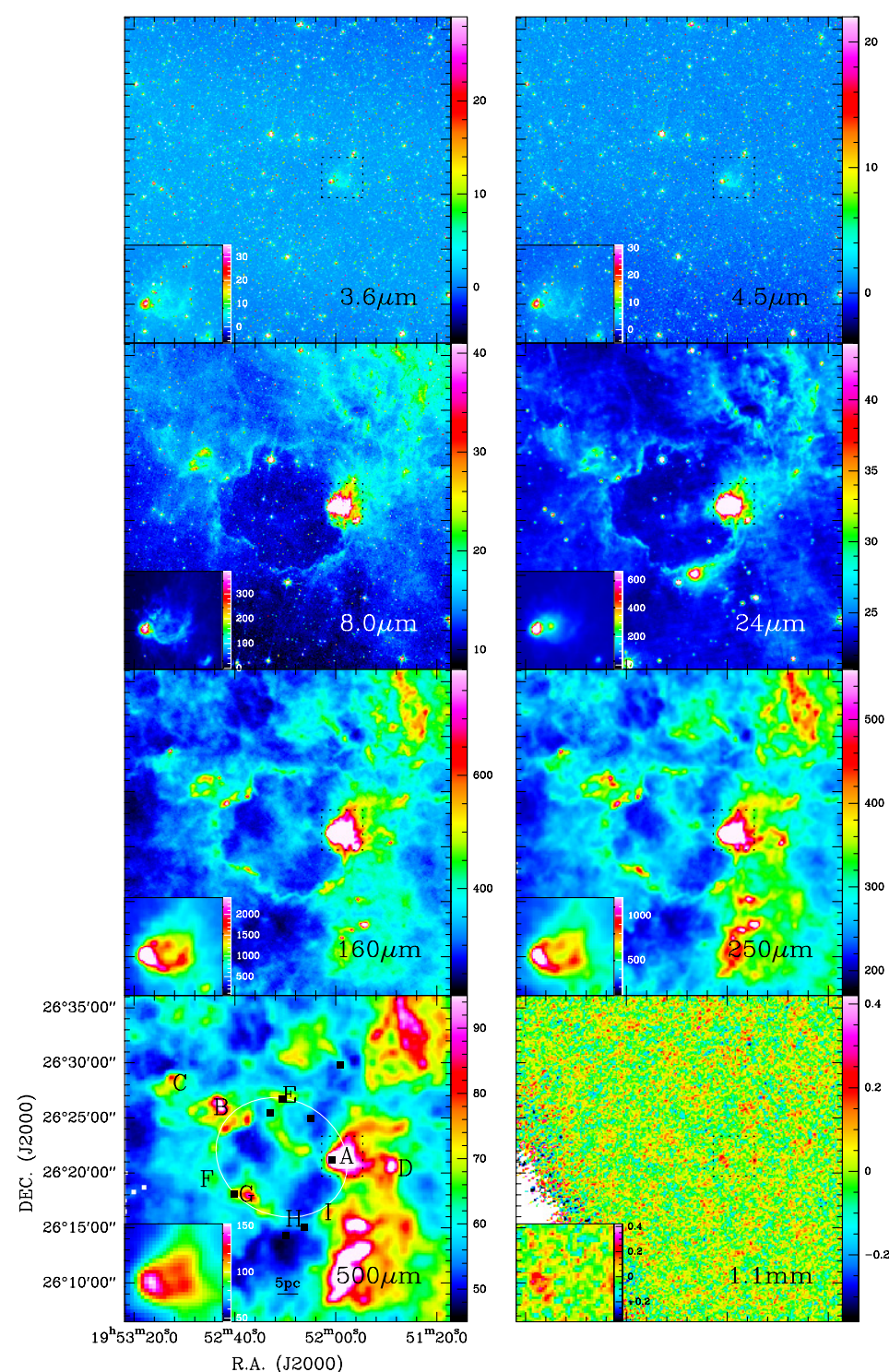

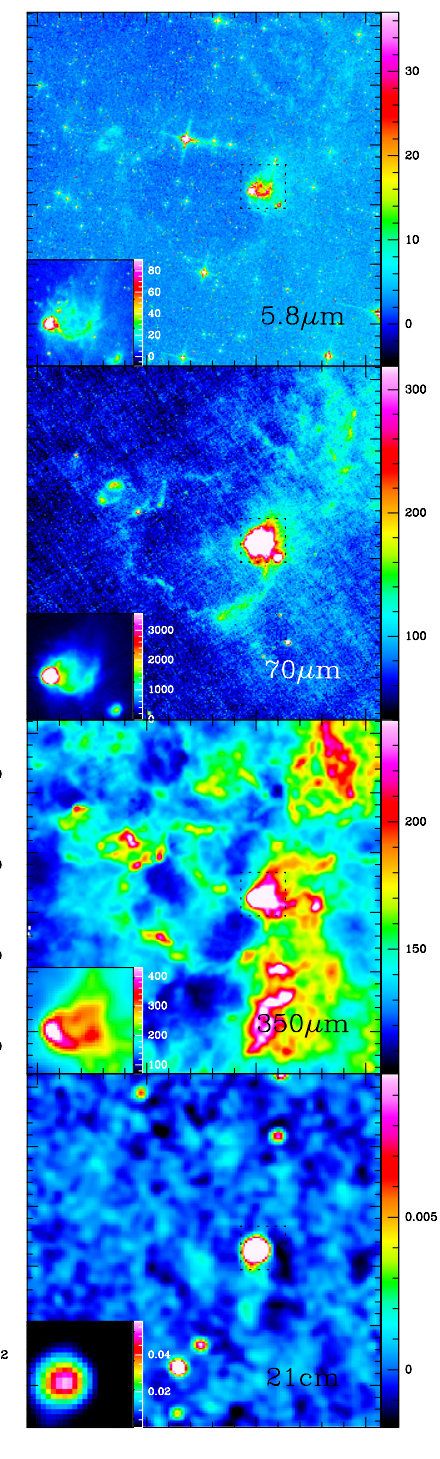

Fig. 2. Multiwavelength images of the bubble N131 from $3.6 \mu \mathrm{m}$ to $21 \mathrm{~cm}$. The saturated region within the dashed square is shown in the bottomleft corner of each subfigure, as a zoom in with adjusted intensity scale. The unit of each colour bar is, respectively, MJy $\mathrm{sr}^{-1}$ for 3.6, 4.5, 5.8, 8.0, 24, $70,160,250,350,500 \mu \mathrm{m}$, Jy beam ${ }^{-1}$ for $1.1 \mathrm{~mm}$, and $21 \mathrm{~cm}$. In the $500 \mu \mathrm{m}$ subfigure, the symbols " $\square$ ", letters (A, B, ..., and I), and ellipse indicate the positions of eight IRAS point sources, nine molecular clumps, and the ring-like shell of the bubble, respectively. outwards. Owing to density differences in pre-existing gas, it is likely that the strong expansion just moved into low densities, but expansion was slowed down at high densities.

In Fig. 3, we present three higher angular resolution integrated intensity maps of $\mathrm{CO}(2-1), \mathrm{CO}(1-0)$, and ${ }^{13} \mathrm{CO}(1-0)$. At large scales, the higher resolution observations have a similar morphology to previous molecular observations. At small scales, there are some intriguing differences between them. Clump A is resolved into several small fragments, which show filamentary structures elongating along the molecular clump (see also Fig. 6 zoomed in). Among the locations of the small fragments within clump A, there is an elongated cavity in the east-west direction. Clump B also breaks up into several small fragments along the northeast-southwest direction.

\subsection{Comparison between dust and gas clumps}

The molecular CO emission in Fig. 3 is correlated with the cold dust emission distribution in Fig. 2 (see also Fig. 2 in Zhang et al. 2013). Between clumps A and E, the ring-like shells open towards the north-west direction where no dust and gas emission has been detected, while two IRAS point sources are detected nearby. Towards clumps C, D, and F, the dust emission is relatively weak; however, their $\mathrm{CO}$ emission is stronger. We suggest that the clumps $\mathrm{C}, \mathrm{D}$, and $\mathrm{F}$ resemble infrared dark clouds (IRDCs; e.g. Rathborne et al. 2006; Simon et al. 2006). The three clumps are relatively far away from the bubble centre. Furthermore, the ring-like shells of the $\mathrm{CO}$ emission have a bright inner edge and a dark outer edge of dust emission.

The IRAS point source $19499+2613$ is close to the eastern part of the secondary bubble N131-A, and is located at the head position of cometary clump A. As seen in Fig. 3, like the dust emission, the $\mathrm{CO}$ emission is extended to the west, although on a much larger scale. The cometary tails are the shell of the secondary bubble N131-A (see Fig. 6). However, the material in this shell might have been partly influenced by the strong stellar winds of N131.

\subsection{Flux distribution on the ring-like shell}

Figure 3 shows a good correlation between the $24 \mu \mathrm{m}$ and $\mathrm{CO}$ (1-0) morphology. Slice profiles of the $24 \mu \mathrm{m}$ and the CO (1-0) emission are shown in Fig. 4 through the clumps AD, E, F, and $\mathrm{G}$. We found a steep rise at the inner edge of the bubble and a gradual fall at the outer edge of the bubble. This steep rise indicates that the inner part of the bubble has been compressed by 


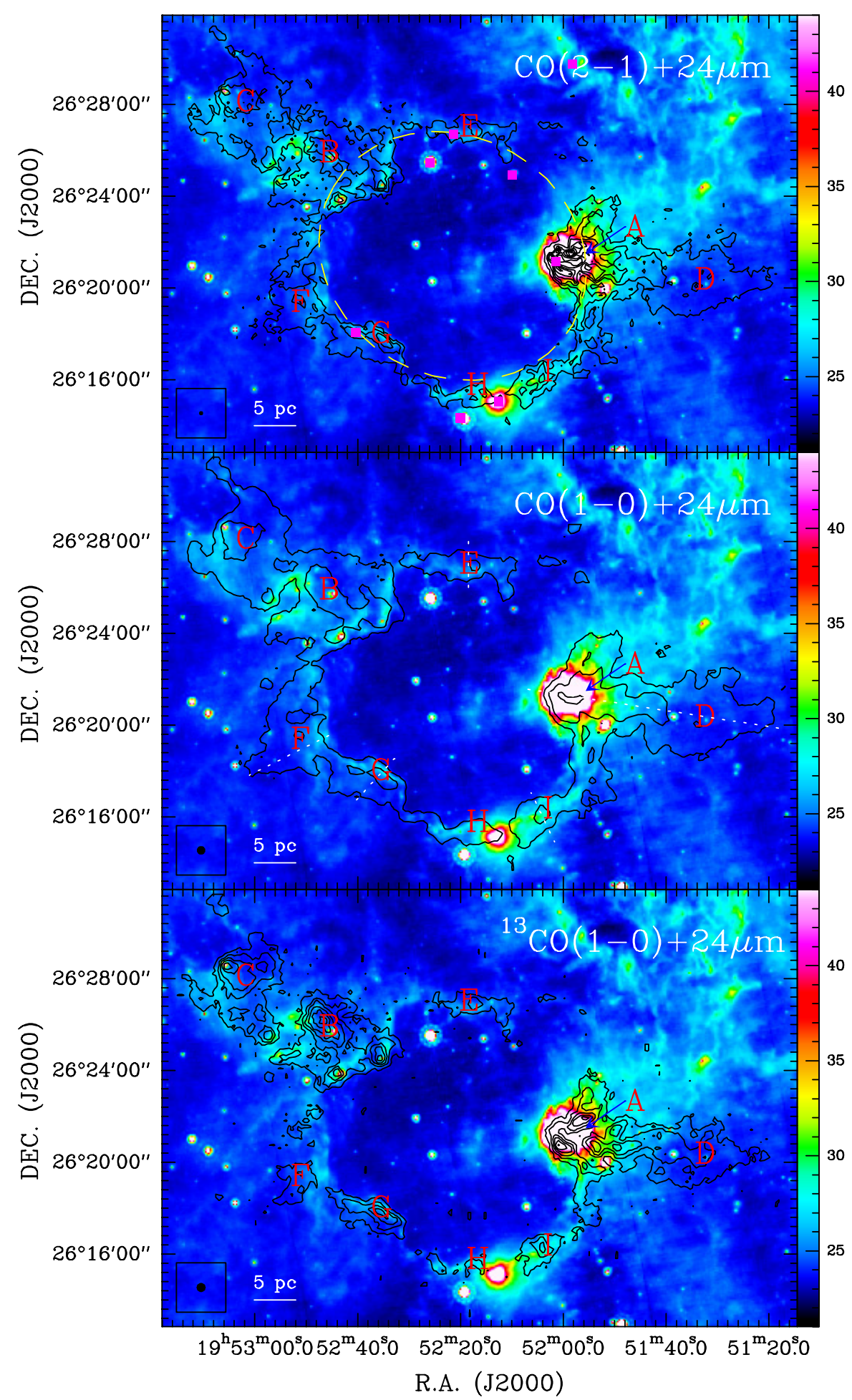

Fig. 3. Integrated intensity maps of the CO (2-1) (upper), CO (1-0) (middle), and ${ }^{13} \mathrm{CO}(1-0)$ (lower) lines with velocity range from -14.5 to $-6.5 \mathrm{~km} \mathrm{~s}^{-1}$ superimposed on $24 \mu \mathrm{m}$ emission. The contour levels start are at $3 \sigma$ in steps of $5 \sigma$ for CO $(2-1)\left(\sigma=1.7 \mathrm{~K}\left(T_{\mathrm{A}}^{*}\right) \mathrm{km} \mathrm{s}^{-1}\right), \mathrm{CO}(1-0)$ $\left(\sigma=1.5 \mathrm{~K}\left(T_{\mathrm{A}}^{*}\right) \mathrm{km} \mathrm{s}^{-1}\right)$, and ${ }^{13} \mathrm{CO}(1-0)\left(\sigma=0.4 \mathrm{~K}\left(T_{\mathrm{A}}^{*}\right) \mathrm{km} \mathrm{s}^{-1}\right)$. The beam size of each subfigure is indicated at the bottom-left corner. The symbols "ø", letters (A, B, ..., and I), and ellipse indicate the positions of eight IRAS point sources, nine molecular clumps, and ring-like shell of the bubble, respectively. The unit of each colour bar is in $\mathrm{MJy} \mathrm{sr}^{-1}$. The dotted lines show the cutting direction of the slice profiles in Fig. 4 and the PV diagram in Fig. 5.

the expansion from the stellar wind. Bubble N131 is very hollow, and most of the material within the bubble has been likely transported onto the ring-like shell. The gradual fall indicates that outside the bubble the molecular gas is pre-existing and that there has been little interaction with the stellar winds within the bubble.

\subsection{Position-velocity diagram}

In Fig. 5, we show four CO (1-0) position-velocity (PV) diagrams for the cuts that we defined in Fig. 4. The PV diagram can be used to identify a possible 3D structure of the bubble: an expanding shell should give rise to an arc-shaped structure in the 

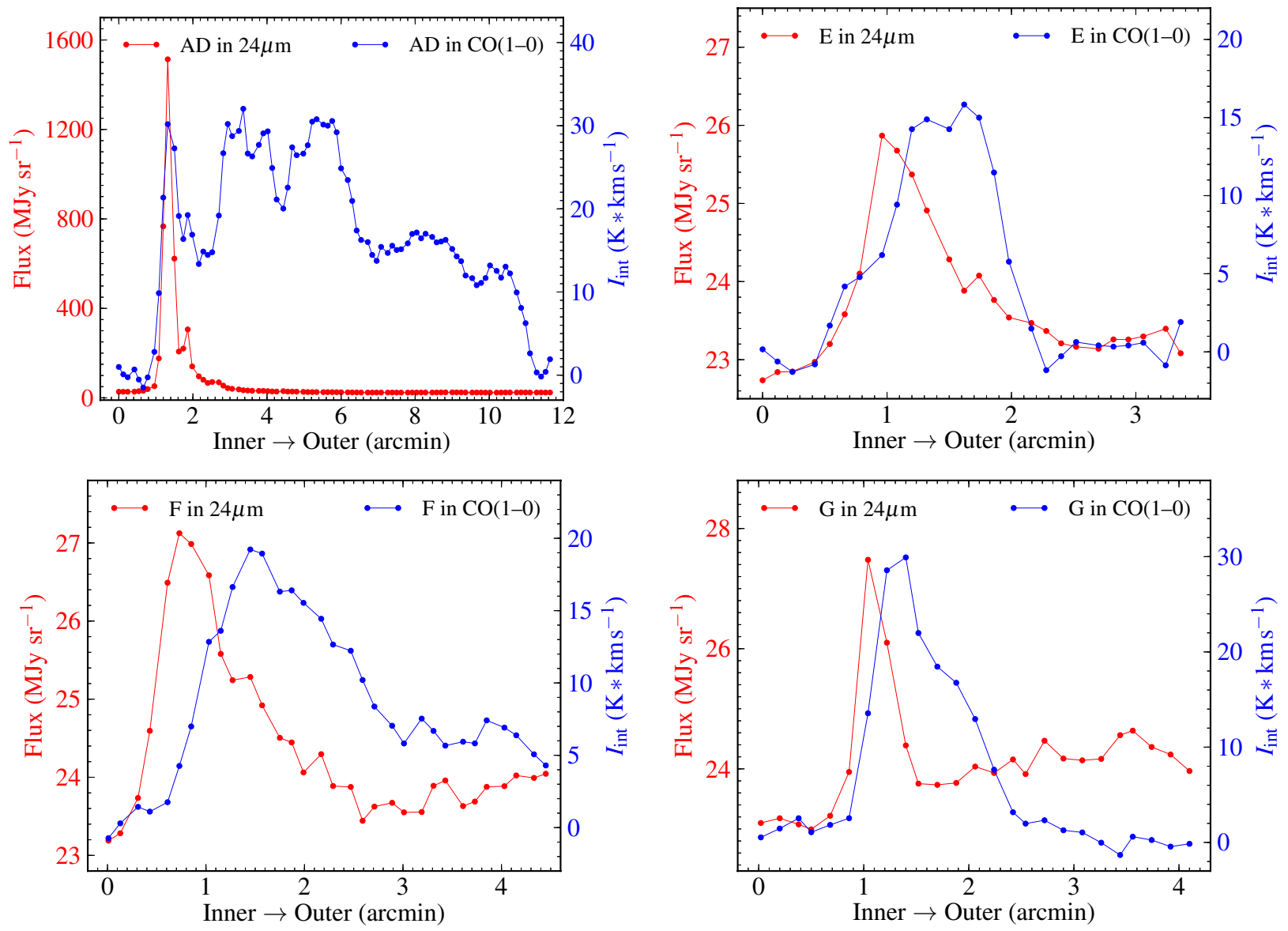

Fig. 4. Slice profiles of $24 \mu \mathrm{m}$ (red) and CO (1-0) emission (blue) from the inner edge to the outer edge of the ring-like shell through the clumps AD, E, F, and G. The cutting paths are shown in Fig. 3. The profiles show a distribution of steep rise at the inner edge and a gradual fall at the outer edge.

PV space (Peng et al. 2010). The PV diagrams do not show obviously arc-shaped structures; however, the inner edge has larger velocity dispersion than the outer edge of the ring-like shell.

In contrast, around clump A, the $\mathrm{CO}$ emission clearly exhibits a 3D structure with the front side and a back side of an expanding ring-like shell, which actually are the front and back sides of the secondary bubble N131-A (see details in Sect. 3.7).

\subsection{Secondary bubble N131-A}

In the previous sections, we introduced a newly found secondary bubble N131-A centred at $\alpha(\mathrm{J} 2000)=19^{\mathrm{h}} 52^{\mathrm{m}} 00^{\mathrm{s}} .10$ and $\delta(\mathrm{J} 2000)=26^{\circ} 21^{\prime} 16^{\prime \prime}{ }^{\prime} 1$, which is located at the position of clump A (see the zoomed-in square in Fig. 2). Figure 6 also shows the N131-A structure. The colour scale for each panel is a SpitzerIRAC RGB image with $4.5 \mu \mathrm{m}$ in blue, $8 \mu \mathrm{m}$ in green, and $24 \mu \mathrm{m}$ in red. The contours in the left and right panels are from NVSS $21 \mathrm{~cm}$ continuum and CO (1-0) emission, respectively. Seen from the left panel, the extended $8 \mu \mathrm{m}$ is surrounding the compact $24 \mu \mathrm{m}$ emission, and the peak of the $21 \mathrm{~cm}$ continuum is located at the peak of the compact $24 \mu \mathrm{m}$ emission. Seen from the CO (1-0) emission three fragments, numbers 1,2 , and 6 (see Table 1 in Sect. 3.2), shaped the shell of the secondary bubble N131-A. In Fig. 5, the PV diagram through clump A clearly shows a 3D structure with a front cloud and a back cloud. Its expanding speed is about $5.5 \mathrm{~km} \mathrm{~s}^{-1}$. Therefore, the secondary bubble N131-A is a typical bubble with an expanding H II region (Churchwell et al. 2006).

Furthermore, we searched for the NVSS catalogue and obtained a total flux of $S_{v}=152.3 \mathrm{mJy}$ at $v=1.4 \mathrm{GHz}$ in the secondary bubble N131-A (Condon et al. 1998). The flux of stellar Lyman photons $N_{\text {LyC }}$ absorbed by the gas in the $\mathrm{H}$ II region can be computed following the relation (Mezger et al. 1974)

$\left(\frac{N_{\mathrm{LyC}}}{\mathrm{s}^{-1}}\right)=\frac{4.761 \times 10^{48}}{a\left(v, T_{\mathrm{e}}\right)}\left(\frac{v}{\mathrm{GHz}}\right)^{0.1}\left(\frac{T_{\mathrm{e}}}{\mathrm{K}}\right)^{-0.45}\left(\frac{S_{v}}{\mathrm{Jy}}\right)\left(\frac{D}{\mathrm{kpc}}\right)^{2}$,

where $a\left(v, T_{\mathrm{e}}\right) \sim 1$ is a slowly varying function tabulated by Mezger \& Henderson (1967), the effective temperature of the central star is assumed to be $T_{\mathrm{e}} \sim 33000 \mathrm{~K}$, and the distance is $D=8.6 \mathrm{kpc}$. The power exponent of $T_{\mathrm{e}}$ is small, so the result does not depend strongly on the chosen $T_{\mathrm{e}}$. Based on the above, we derived a Lyman-continuum ionizing photon flux of $\log \left(N_{\text {LyC }}\right)=\sim 47.7$ from the $\mathrm{H}$ II region, which is equivalent to an O9.5 star (Panagia 1973).

\subsection{Moments 1 and 2}

The velocity structure (moment 1) of bubble N131 has been analysed in Figs. 4-6 of Zhang et al. (2013), and in Figs. 5 and 7 of this work. Along the central axis of the clumps AD and BC, there is an apparent velocity gradient. At the systematic velocity of $-10.5 \mathrm{~km} \mathrm{~s}^{-1}$, clump $\mathrm{AD}$ is redshifted with a velocity range of [ $-9.5-6.5] \mathrm{km} \mathrm{s}^{-1}$, while clump BC is blueshifted with a velocity range of $[-14.5-11.5] \mathrm{km} \mathrm{s}^{-1}$. This indicates that clumps 
C.-P. Zhang et al.: The infrared dust bubble N131
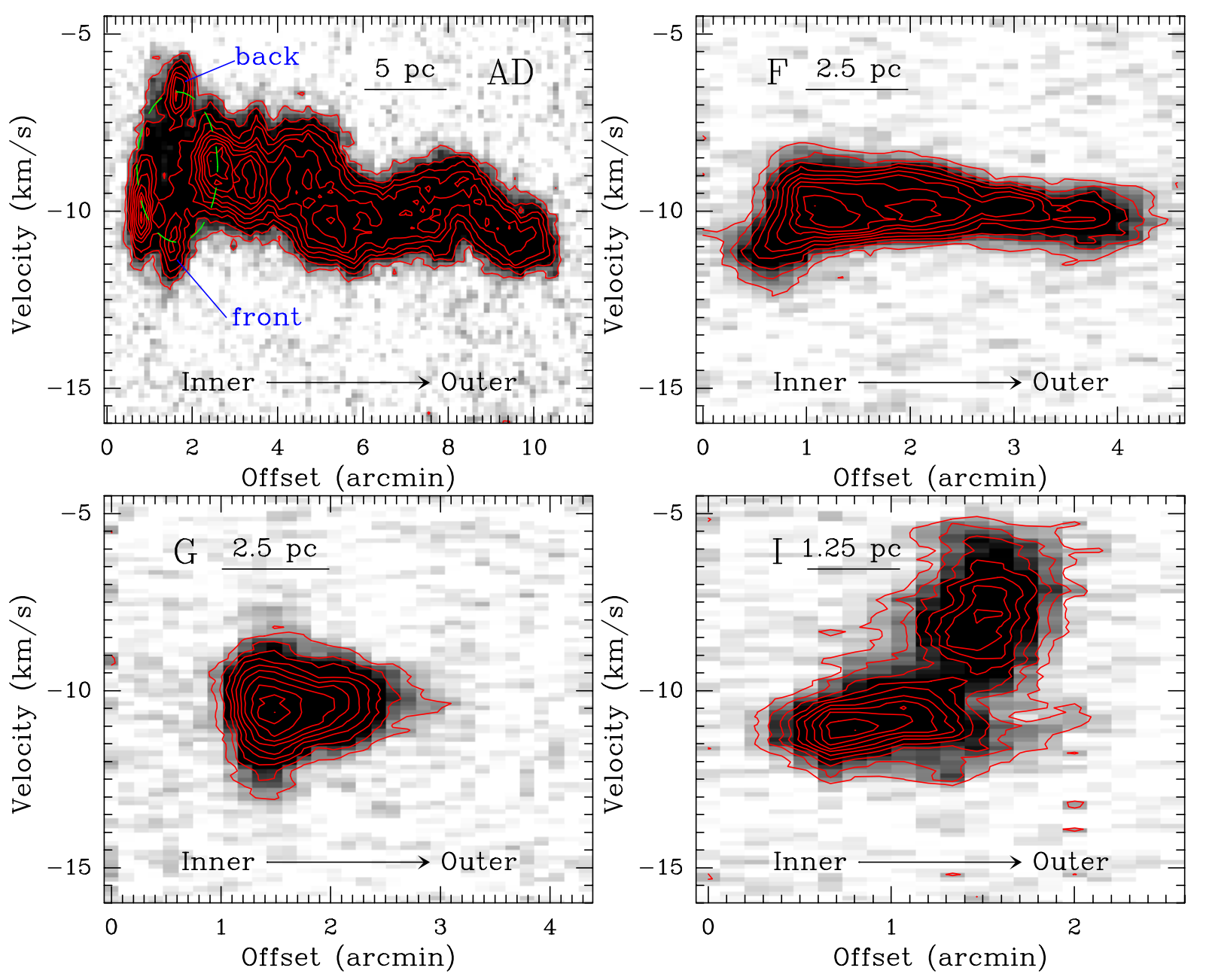

Fig. 5. Position-velocity diagram from the inner edge to the outer edge of the ring-like shell through the clumps AD, E, F, and I for the CO (1-0). The cutting paths are shown in Fig. 3. The green dashed ellipse indicates the 3D structure of the secondary bubble N131-A with front and back sides. Contour levels start at 3 times the rms level of $1.5 \mathrm{~K}$ and increase in steps of $1.5 \mathrm{~K}$.
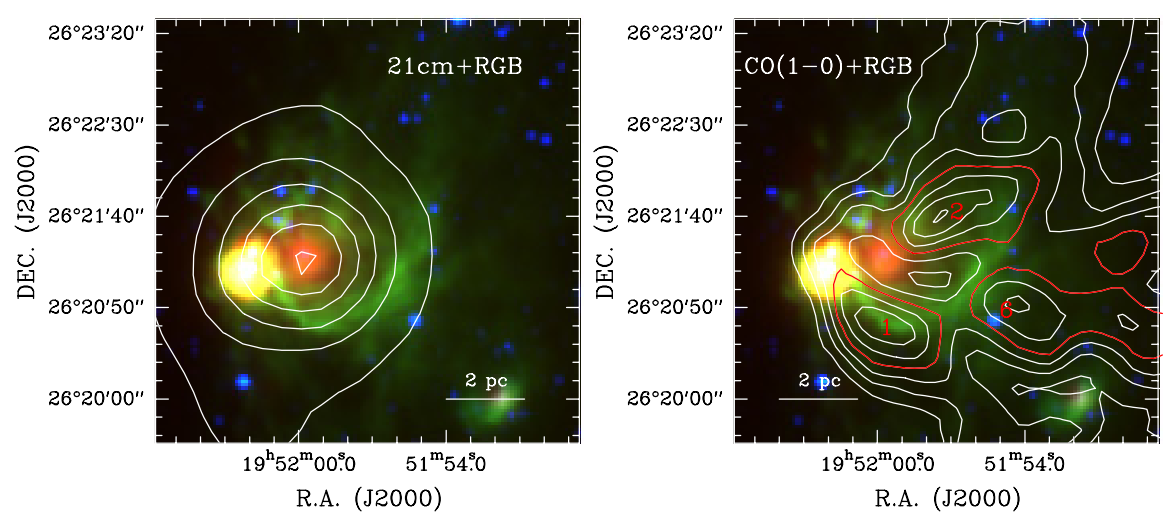

Fig. 6. NVSS $21 \mathrm{~cm}$ continuum (left) and IRAM $30 \mathrm{~m}$ CO (1-0) (right) contours overlaid on a Spitzer-IRAC three-colour image of the secondary bubble N131-A with $4.5 \mu \mathrm{m}=$ blue, $8 \mu \mathrm{m}=$ green, and $24 \mu \mathrm{m}=$ red. The contour levels start at $5 \sigma$ in steps of $20 \sigma$ with $\sigma=$ $0.53 \mathrm{mJy}$ beam $^{-1}$ for the $21 \mathrm{~cm}$, and at $5 \sigma$ in steps of $5 \sigma$ with $\sigma=1.5 \mathrm{~K}\left(T_{\mathrm{A}}^{*}\right) \mathrm{km} \mathrm{s}^{-1}$ for the $\mathrm{CO}(1-0)$. The numbers 1,2 , and 6 indicate fragment positions from Table 1 . These two maps are taken from the zoom-in in the dashed square of Fig. 2.

$\mathrm{AD}$ and $\mathrm{BC}$ are two filamentary flows transferring material outwardly, probably owing to the strong stellar wind. In addition, clumps $\mathrm{G}$ and $\mathrm{H}$ in the ring-like shell are blueshifted and clumps $\mathrm{E}$ and I are redshifted, which indicates that the ring-like shell of the bubble is expanding outwardly.

For clump D in Fig. 7, in addition to the velocity gradient in its east-west direction, there is another velocity gradient in its north-south direction. Clump $\mathrm{F}$ has a velocity component similar to the systematic velocity of $-10.5 \mathrm{~km} \mathrm{~s}^{-1}$. This can be explained if the expansion was blocked off by a pre-existing clump.
In the lower part of Fig. 7, we present the velocity dispersion (moment 2) distribution of bubble N131 determined from our CO (1-0) data. The maximum is $4.4 \mathrm{~km} \mathrm{~s}^{-1}$ at the secondary bubble N131-A. The broadening is due to observing emission from both the front and the back sides along this sight line. At clumps B and I, the maximum is 3.4 and $4.0 \mathrm{~km} \mathrm{~s}^{-1}$, respectively; at other clumps, the velocity dispersion ranges from 1.2 to $2.2 \mathrm{~km} \mathrm{~s}^{-1}$. Overall, the velocity dispersion decreases from the inner edge to outer edge of the ring-like shell, which is consistent with the PV diagram in Fig. 5. 
Table 1. Parameters of fragments determined from Gaussclumps analysis.

\begin{tabular}{|c|c|c|c|c|c|c|c|c|c|c|c|c|}
\hline \multirow{2}{*}{$\begin{array}{l}\text { No. } \\
\text { (1) }\end{array}$} & \multirow{2}{*}{$\begin{array}{l}\text { Offsets } \\
\text { ", ," } \\
(2)\end{array}$} & \multirow{2}{*}{$\begin{array}{c}\text { Gaussian size } \\
\text { " } \times " \\
(3) \\
\end{array}$} & \multicolumn{2}{|c|}{$F W H M$} & \multicolumn{2}{|c|}{ Deconv. FWHM } & \multirow{2}{*}{$\begin{array}{c}N_{\mathrm{H}_{2}} \\
10^{21} \mathrm{~cm}^{-2} \\
(8)\end{array}$} & \multirow{2}{*}{$\begin{array}{c}M \\
M_{\odot} \\
(9) \\
\end{array}$} & \multirow{2}{*}{$\begin{array}{c}V_{\mathrm{lsr}} \\
\mathrm{km} \mathrm{s}^{-1} \\
(10) \\
\end{array}$} & \multirow{2}{*}{$\begin{array}{c}\Delta V \\
\mathrm{~km} \mathrm{~s}^{-1} \\
(11)\end{array}$} & \multirow{2}{*}{$\begin{array}{c}M_{\text {vir }} \\
M_{\odot} \\
(12) \\
\end{array}$} & \multirow{2}{*}{$\begin{array}{l}\alpha_{\mathrm{vir}} \\
\text { (13) } \\
\end{array}$} \\
\hline & & & $\begin{array}{c}\prime \prime \\
(4)\end{array}$ & $\begin{array}{l}\mathrm{pc} \\
(5)\end{array}$ & $\begin{array}{l}\prime \prime \\
(6)\end{array}$ & $\begin{array}{l}\mathrm{pc} \\
(7) \\
\end{array}$ & & & & & & \\
\hline 1 & $(68.8,-95.9)$ & $30.8 \times 61.2$ & 38.9 & 1.6 & 31.7 & 1.3 & 21.2 & 894 & $-9.98(0.01)$ & $1.60(0.02)$ & 521 & 0.58 \\
\hline 2 & $(13.0,-31.5)$ & $65.5 \times 42.5$ & 50.4 & 2.1 & 45.1 & 1.9 & 16.3 & 1160 & $-9.10(0.02)$ & $2.30(0.03)$ & 1401 & 1.21 \\
\hline 3 & $(541.2,128.8)$ & $53.3 \times 37.1$ & 43.0 & 1.8 & 36.7 & 1.5 & 15.5 & 800 & $-11.50(0.01)$ & $1.68(0.03)$ & 636 & 0.80 \\
\hline 4 & $(685.1,224.0)$ & $71.3 \times 132.1$ & 88.7 & 3.7 & 85.8 & 3.6 & 14.9 & 3267 & $-12.28(0.01)$ & $1.90(0.03)$ & 1684 & 0.52 \\
\hline 5 & $(948.3,368.2)$ & $46.2 \times 71.1$ & 54.8 & 2.3 & 49.9 & 2.1 & 14.5 & 1217 & $-12.87(0.01)$ & $1.68(0.03)$ & 811 & 0.67 \\
\hline 6 & $(-11.4,-88.1)$ & $82.8 \times 37.4$ & 48.2 & 2.0 & 42.6 & 1.8 & 13.4 & 872 & $-8.98(0.01)$ & $1.59(0.03)$ & 637 & 0.73 \\
\hline 7 & $(660.9,88.5)$ & $33.6 \times 70.1$ & 42.9 & 1.8 & 36.5 & 1.5 & 13.3 & 682 & $-11.42(0.01)$ & $1.39(0.03)$ & 436 & 0.64 \\
\hline 8 & $(837.0,192.5)$ & $36.7 \times 41.4$ & 38.8 & 1.6 & 31.6 & 1.3 & 12.3 & 519 & $-12.28(0.01)$ & $1.01(0.02)$ & 207 & 0.40 \\
\hline 9 & $(-75.3,-103.8)$ & $28.8 \times 68.2$ & 40.0 & 1.7 & 33.0 & 1.4 & 12.0 & 538 & $-9.21(0.02)$ & $2.23(0.04)$ & 1042 & 1.94 \\
\hline 10 & $(549.0,-271.9)$ & $37.3 \times 116.6$ & 50.2 & 2.1 & 44.9 & 1.9 & 11.1 & 779 & $-10.73(0.01)$ & $1.35(0.02)$ & 478 & 0.61 \\
\hline 11 & $(-67.2,-55.6)$ & $36.8 \times 25.6$ & 29.7 & 1.2 & 19.4 & 0.8 & 10.1 & 251 & $-8.88(0.02)$ & $1.98(0.04)$ & 613 & 2.45 \\
\hline 12 & $(117.0,-367.6)$ & $54.1 \times 53.2$ & 53.6 & 2.2 & 48.7 & 2.0 & 8.1 & 648 & $-7.70(0.02)$ & $1.78(0.05)$ & 890 & 1.37 \\
\hline 13 & $(845.3,360.4)$ & $72.7 \times 107.7$ & 88.5 & 3.7 & 85.6 & 3.6 & 8.0 & 1747 & $-11.74(0.02)$ & $1.69(0.04)$ & 1324 & 0.76 \\
\hline 14 & $(4.9,-151.8)$ & $44.5 \times 22.7$ & 28.6 & 1.2 & 17.7 & 0.7 & 7.5 & 171 & $-9.60(0.01)$ & $1.24(0.03)$ & 230 & 1.34 \\
\hline 15 & $(4.9,32.4)$ & $39.1 \times 65.6$ & 47.5 & 2.0 & 41.8 & 1.7 & 7.2 & 455 & $-8.81(0.01)$ & $1.23(0.03)$ & 380 & 0.84 \\
\hline 16 & $(285.0,272.3)$ & $163.4 \times 45.0$ & 61.3 & 2.6 & 57.0 & 2.4 & 7.0 & 737 & $-8.51(0.04)$ & $1.72(0.08)$ & 958 & 1.30 \\
\hline 17 & $(-203.3,-119.7)$ & $86.7 \times 96.4$ & 91.4 & 3.8 & 88.6 & 3.7 & 6.9 & 1618 & $-10.42(0.02)$ & $1.71(0.05)$ & 1409 & 0.87 \\
\hline 18 & $(541.0,168.4)$ & $38.4 \times 104.7$ & 52.2 & 2.2 & 47.1 & 2.0 & 6.5 & 497 & $-11.02(0.03)$ & $1.95(0.06)$ & 1046 & 2.11 \\
\hline 19 & $(780.9,136.3)$ & $94.3 \times 45.0$ & 57.4 & 2.4 & 52.8 & 2.2 & 6.3 & 577 & $-11.26(0.01)$ & $0.88(0.04)$ & 232 & 0.40 \\
\hline 20 & $(-35.0,-159.7)$ & $22.5 \times 43.2$ & 28.2 & 1.2 & 17.0 & 0.7 & 6.2 & 137 & $-9.27(0.01)$ & $1.10(0.03)$ & 179 & 1.30 \\
\hline 21 & $(612.0,124.4)$ & $53.5 \times 79.4$ & 30.1 & 1.3 & 19.9 & 0.8 & 5.8 & 146 & $-10.99(0.02)$ & $1.26(0.04)$ & 252 & 1.73 \\
\hline 22 & $(861.0,248.3)$ & $69.1 \times 46.2$ & 54.3 & 2.3 & 49.4 & 2.1 & 5.1 & 423 & $-11.62(0.02)$ & $1.40(0.04)$ & 561 & 1.33 \\
\hline 23 & $(-99.1,-39.6)$ & $37.9 \times 28.7$ & 32.4 & 1.4 & 23.3 & 1.0 & 5.0 & 145 & $-8.43(0.03)$ & $1.85(0.09)$ & 580 & 3.98 \\
\hline 24 & $(-307.1,-127.6)$ & $42.6 \times 150.4$ & 58.0 & 2.4 & 53.4 & 2.2 & 4.9 & 461 & $-10.04(0.02)$ & $1.66(0.04)$ & 843 & 1.83 \\
\hline 25 & $(-11.0,-279.6)$ & $98.6 \times 64.4$ & 79.7 & 3.3 & 76.5 & 3.2 & 4.8 & 858 & $-10.38(0.05)$ & $1.95(0.11)$ & 1591 & 1.85 \\
\hline 26 & $(749.0,-175.7)$ & $49.3 \times 70.9$ & 57.1 & 2.4 & 52.5 & 2.2 & 4.6 & 423 & $-9.89(0.03)$ & $1.51(0.07)$ & 688 & 1.62 \\
\hline 27 & $(1013.0,288.3)$ & $65.7 \times 69.9$ & 65.8 & 2.7 & 61.8 & 2.6 & 4.4 & 530 & $-12.95(0.02)$ & $1.33(0.06)$ & 615 & 1.16 \\
\hline 28 & $(-371.1,-127.7)$ & $43.7 \times 92.2$ & 60.2 & 2.5 & 55.8 & 2.3 & 4.3 & 433 & $-9.96(0.04)$ & $1.64(0.08)$ & 854 & 1.97 \\
\hline 29 & $(924.9,312.4)$ & $25.1 \times 96.0$ & 35.2 & 1.5 & 27.0 & 1.1 & 4.2 & 145 & $-11.89(0.02)$ & $1.43(0.04)$ & 379 & 2.62 \\
\hline 30 & $(588.9,208.4)$ & $39.6 \times 47.9$ & 43.1 & 1.8 & 36.8 & 1.5 & 4.1 & 212 & $-11.90(0.04)$ & $1.45(0.10)$ & 476 & 2.24 \\
\hline 31 & $(636.9,272.4)$ & $44.0 \times 23.7$ & 29.5 & 1.2 & 19.1 & 0.8 & 3.8 & 92 & $-12.44(0.02)$ & $0.99(0.04)$ & 151 & 1.64 \\
\hline
\end{tabular}

Notes. Column (1) lists the Gaussian fragment number. Column (2) lists the position offsets from absolute coordinate $\alpha(J 2000)=19^{\mathrm{h}} 51^{\mathrm{m}} 55^{\mathrm{s}} \cdot 212$ and $\delta(J 2000)=26^{\circ} 22^{\prime} 21^{\prime \prime} 04$. Column (3) lists the Gaussian size derived from Gaussclumps. Columns (4)-(7) list the FWHM and deconvolved FWHM. Column (8) lists the extracted peak column density using Gaussclumps. Column (9) lists the obtained fragment masses. Columns (10)(11) list the velocities and line widths from Gaussian fit of ${ }^{13} \mathrm{CO}(1-0)$. Column (12) lists the virial mass. And Col. (13) lists the virial parameters $\alpha_{\mathrm{vir}}=M_{\mathrm{vir}} / M$.

\subsection{Excitation temperature and column density}

Assuming that the lower transitions of the CO molecule are under local thermal equilibrium (LTE), their excitation temperature and column density can be computed (Winnewisser et al. 1979; Garden et al. 1991). The CO (1-0) line is assumed to be optically thick, with a beam-filling factor of unity. Assuming that the excitation temperature and kinematic temperature for $\mathrm{CO}(1-0)$ are the same for ${ }^{13} \mathrm{CO}(1-0)$, the excitation temperatures $T_{\text {ex }}$ and then the column density $N_{13} \mathrm{CO}$ can be calculated directly using conversion factors of $\left[\mathrm{H}_{2}\right] /\left[{ }^{12} \mathrm{CO}\right]=10^{4}$ and $[\mathrm{CO}] /\left[{ }^{13} \mathrm{CO}\right]=60$. The obtained $T_{\mathrm{ex}}$ and $N_{\mathrm{H}_{2}}$ maps are shown in Fig. 8.

In the upper part of Fig. 8, $T_{\mathrm{ex}}$ mainly ranges from 9.1 to $16.5 \mathrm{~K}$ in the diffuse structure, while excitation temperatures between 16.5 and $30.6 \mathrm{~K}$ are found towards several compact fragments. The higher $T_{\mathrm{ex}}$ values are generally found at the inner edge of the ring-like shell whose temperature decreases from the inside to the outside. We suggest that the heating is caused by warm stellar winds within the bubble.

In the lower part of Fig. 8, $N_{\mathrm{H}_{2}}$ mainly ranges from $4.0 \times$ $10^{21}$ to $14.0 \times 10^{21} \mathrm{~cm}^{-2}$, and the maximum is $21.4 \times 10^{21} \mathrm{~cm}^{-2}$ located at fragment No.1 (see Table 1) in clump A. The denser regions are distributed at clumps $\mathrm{AD}$ and $\mathrm{BC}$, while the ring-like shell of the bubble N131 is very diffuse and just has few dense fragments. Furthermore, clump A is denser than clump D, and clump B is as dense as clump C. These clumps have fragmented into several dense fragments, which is discussed in Sect. 4.2.

The ${ }^{13} \mathrm{CO}$ isotope probes only relatively low volume densities, and may be depleted in fragments with high densities. It will lead to underestimating the densities and masses of the fragments. Alternatively, the dust continuum emission can be used to derive column densities, but based on the CO observations, there are several velocity components found on the line of sight towards the N131 which continuum data cannot distinguish. In Fig. 2 it is obvious that the Herschel data is in general not well correlated with the ${ }^{13} \mathrm{CO}$ emission. However, several of the fragments show clear counterparts in the Herschel images, and so we combined 70,160 , and $250 \mu \mathrm{m}$ data to estimate the column densities (Preibisch et al. 2012), which are found to be comparable to the column densities from ${ }^{13} \mathrm{CO}$ emission. In the following, we therefore use the column densities and masses derived from ${ }^{13} \mathrm{CO}$. 


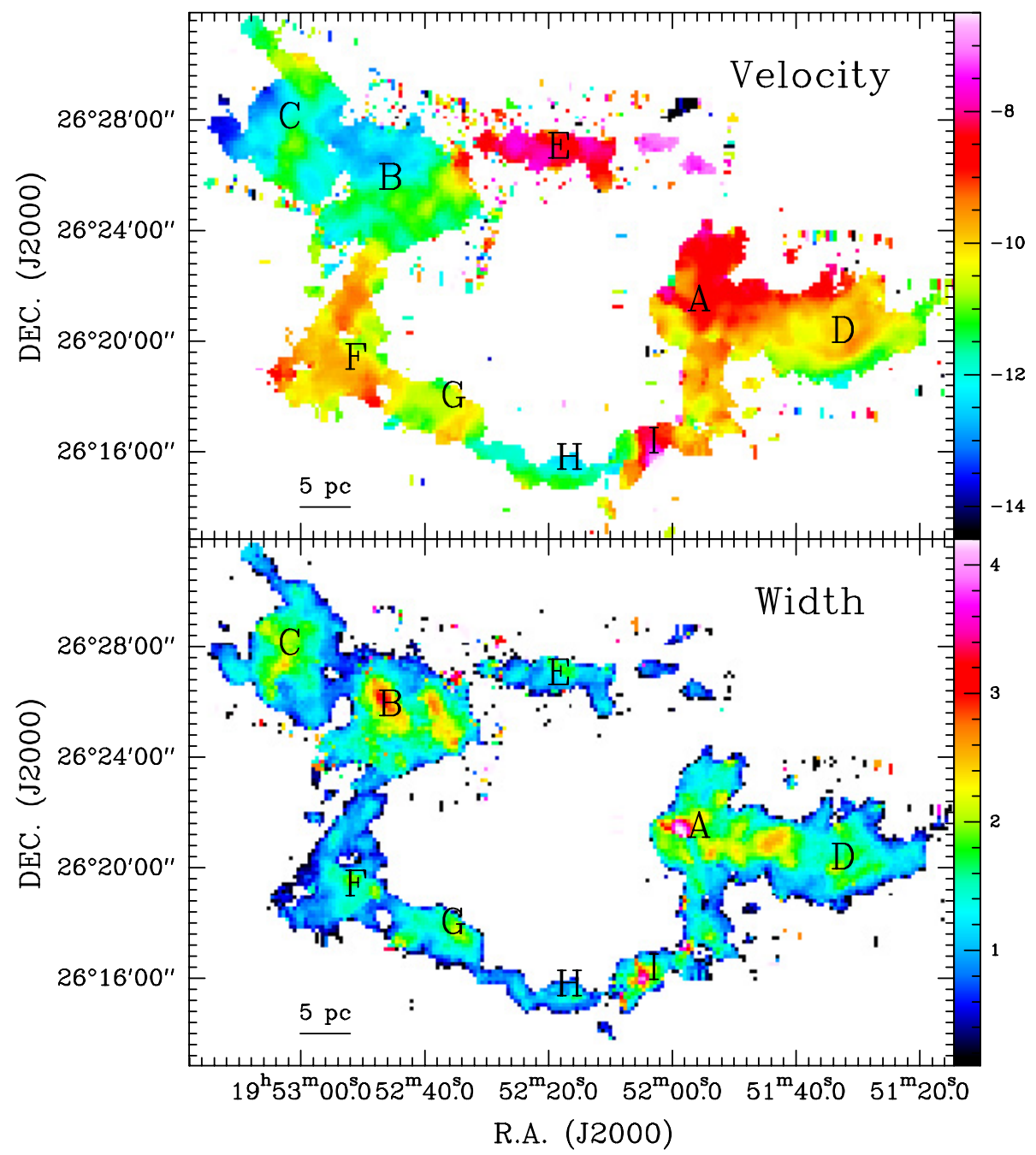

Fig. 7. The intensity-weighted mean velocity map (moment 1 ; upper) and velocity dispersion map (moment 2 ; lower). The unit of each colour bar is $\mathrm{km} \mathrm{s}^{-1}$. The letters from A to I indicate the positions of nine molecular clumps.

\section{Discussion}

\subsection{Stellar wind}

The strong stellar winds from the central OB-type stars, along with overpressure from ionization and high temperature, may cause the expansion of gas/dust shells in their vicinity, of which N131 is an example (Castor et al. 1975; Weaver et al. 1977). In particular, dust emission at $24 \mu \mathrm{m}$ can be used as an indicator of the effects of winds. Very small grains, heated by the stellar radiation and out of thermal equilibrium, radiate at $24 \mu \mathrm{m}$ (Jones et al. 1999). Deharveng et al. (2010) found that $86 \%$ of the bubbles contain ionized gas detected in $20 \mathrm{~cm}$ radio continuum radiation and $98 \%$ of the bubbles exhibit extended and dominated $24 \mu \mathrm{m}$ emission in their central regions. A central hole in $24 \mu \mathrm{m}$ emission maps of some H II regions might be an indication that winds are at work (Watson et al. 2008). Such a hole could also be attributed to the radiation pressure of the central ionizing stars or to dust destruction by the stars' ionizing radiation (Krumholz \& Matzner 2009; Martins et al. 2010) and strong stellar winds (Castor et al. 1975; Weaver et al. 1977).

In the direction of the centre of bubble N131, we detect very weak $21 \mathrm{~cm}$ continuum and $24 \mu \mathrm{m}$ emission; it has been suggested that the emission is an unrelated background component, so bubble N131 is a rare sample in a different evolutionary stage (Deharveng et al. 2010). However, strong emission at $24 \mu \mathrm{m}$ was observed in the direction of the dense PDR filaments delineating the ring-like shell of the bubble. The $24 \mu \mathrm{m}$ emission mostly originates in the PDR filament, and may have been compressed by the strong stellar winds emitted by the ionizing stars. This indicates that the material giving rise to the $24 \mu \mathrm{m}$ emission inside N131 cannot survive the intense radiation field produced by the central cluster, and the bubble is likely evacuated of ionized gas and hot dust by the strong stellar winds of early OB-type stars. Therefore, bubble N131 is at an advanced stage of evolution.

\subsection{Fragmentation}

In the lower panel of Fig. 8, we show the Gaussian fragments extracted using RUN GAUSSCLUMPS in the GILDAS software package. The position and size of each fragment is indicated with an ellipse in the column density map of Fig. 8. These fragments are mostly distributed at clumps $\mathrm{AD}$ and $\mathrm{BC}$, and there are only five fragments located at the other clumps. This suggests that the ring-like shell is more diffuse than clumps AD and $\mathrm{BC}$, and fragmentation is more pronounced towards clumps $\mathrm{AD}$ and $\mathrm{BC}$ than in the ring-like shell.

In Fig. 9, we present the mass-size relation diagram for the extracted fragments. Comparison with the high-mass star formation threshold of $m(r)>870 M_{\odot}(r / \mathrm{pc})^{1.33}$ empirically proposed by Kauffmann \& Pillai (2010) allows us to determine whether these fragments are capable of giving birth to massive stars. The data points are distributed below the threshold (given by the red line in Fig. 9) that discriminates between high- and lowmass star formation whose entries fall above and below the line, respectively, indicative of low-mass star-forming candidates. 


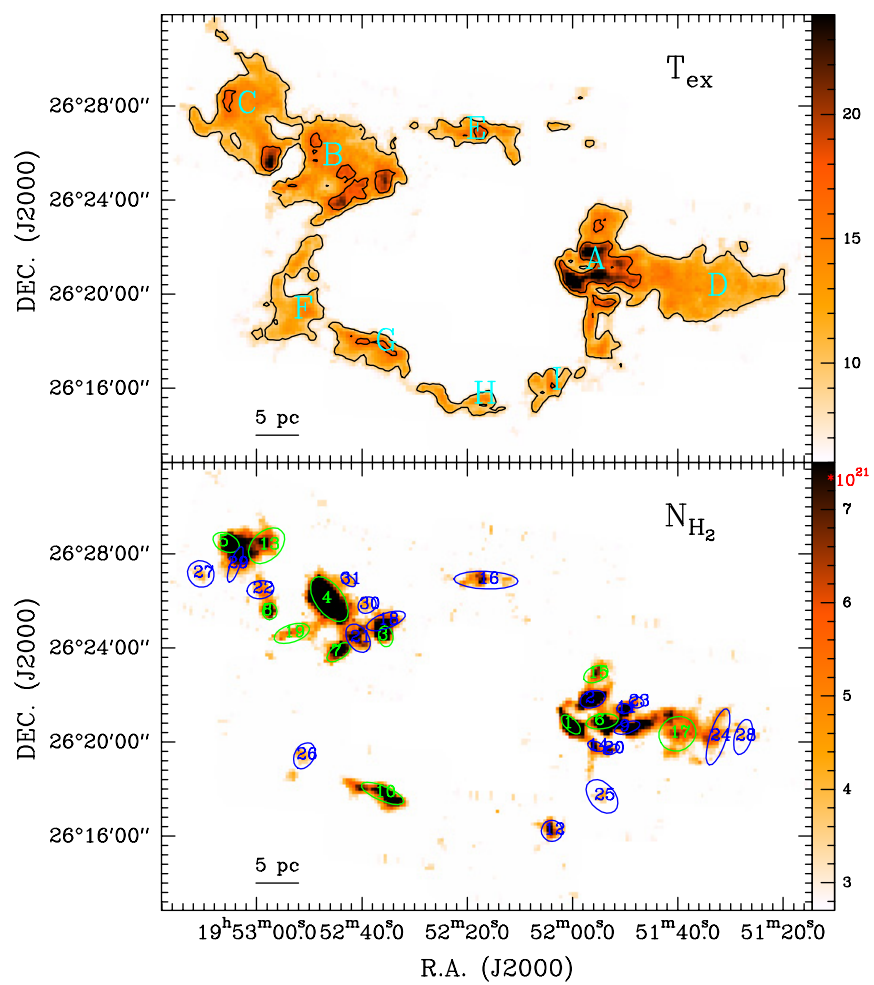

Fig. 8. N131: CO (1-0) excitation temperature map ( $T_{\mathrm{ex}} ;$ upper $)$ and $\mathrm{H}_{2}$ column density distribution $\left(N_{\mathrm{H}_{2}}\right.$; lower $)$. For $T_{\mathrm{ex}}$, the contour levels are 9.1 and $16.5 \mathrm{~K}$, and the maximum is $30.6 \mathrm{~K}$. The ellipses with numbers indicate the position and size of each fragment extracted by Gaussclumps. The letters from A to I indicate the positions of nine molecular clumps. The green and blue ellipses correspond to $\alpha_{\text {vir }}<1$ and $\alpha_{\text {vir }}>1$, respectively. The units for each colour bar are in Kelvin for $T_{\mathrm{ex}}$ and $10^{21} \mathrm{~cm}^{-2}$ for $N_{\mathrm{H}_{2}}$, respectively.

Lee \& Chen (2007) suggested that radiation from massive stars can only trigger the formation of low- and intermediate-mass objects. It appears that the stellar winds in the N131 bubble only triggered the formation of low-mass stars. It is also likely that some of these fragments in the clumps AD and BC were preexisting and were not triggered by the stellar winds.

\subsection{Virial state}

The virial theorem provides a method to test whether a molecular fragment is in a stable state. If external pressure and magnetic fields are ignored, $M_{\mathrm{vir}} \simeq 210 r \Delta V^{2}\left(M_{\odot}\right)$ (Evans 1999), where $r$ is the fragment radius in pc and $\Delta V^{2}$ is the full width at halfmaximum linewidth in $\mathrm{km} \mathrm{s}^{-1}$. Between the virial mass $M_{\mathrm{vir}}$ and the fragment mass $M$, if the virial parameter $\alpha_{\mathrm{vir}}=M_{\mathrm{vir}} / M<1$, the molecular fragment is gravitationally bound, potentially unstable, and collapsing; if $\alpha_{\mathrm{vir}}>1$, the fragment is not gravitationally bound in a stable or expanding state (Hindson et al. 2013).

Kauffmann \& Pillai (2010) found empirically a massive star formation threshold $m(r)=870 M_{\odot}(r / \mathrm{pc})^{1.33}$ for solar neighbourhood clouds. With our velocity-resolved observations, we address its physical origin studying the virial states of the fragments.

The derived virial masses $M_{\mathrm{vir}}$ and virial parameters $\alpha_{\mathrm{vir}}$ are listed in Table 1. We also show entries for the corresponding fragments with $\alpha_{\text {vir }}<1$ (in green) and $\alpha_{\text {vir }}>1$ (in blue) in Figs. 8 and 9 . Of the extracted 31 fragments, 12 fragments are gravitationally bound, and 19 fragments are not gravitationally

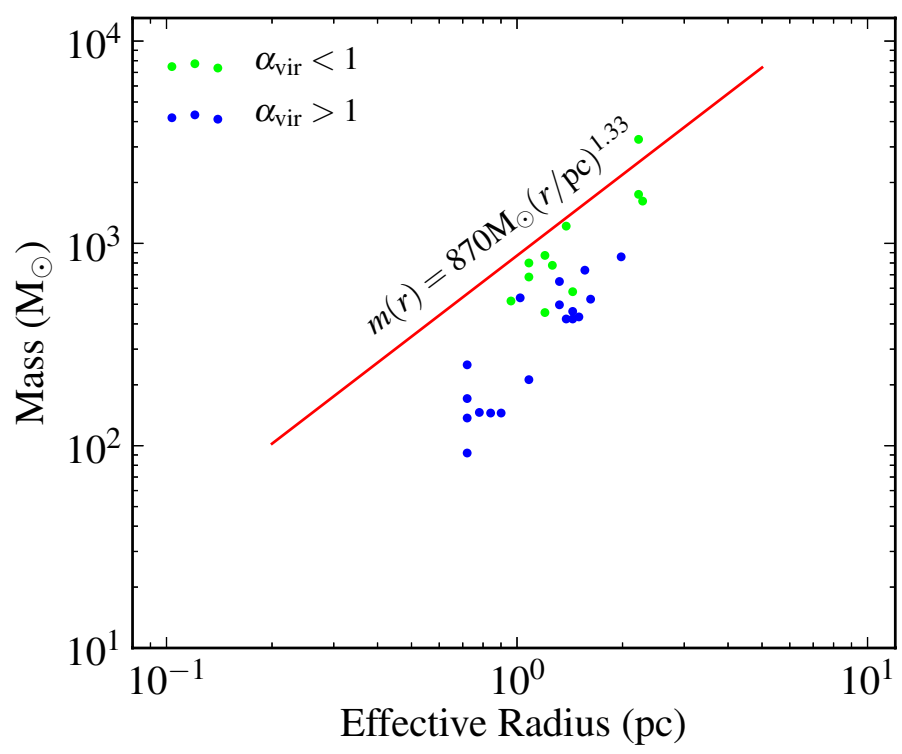

Fig. 9. Mass-radius distributions of Gaussian fragments extracted from Gaussclumps. The masses are listed in Table 1 and the effective radius is derived with $r=F W H M /(2 \sqrt{\ln 2})$. The red line delineates the threshold introduced by Kauffmann \& Pillai (2010) separating the regimes under which high-mass stars can form (above the line) or not (below the line). The green and blue points belong to $\alpha_{\mathrm{vir}}<1$ and $\alpha_{\mathrm{vir}}>1$, respectively.

bound. In Fig. 9, the gravitationally bound and unbound fragments are distributed differently. The gravitationally bound fragments $\left(\alpha_{\text {vir }}<1\right)$ tend to follow the mass-size relation $(m(r)=$ $\left.870 M_{\odot}(r / \mathrm{pc})^{1.33}\right)$ better, and this implies a gravitational origin of the relation. This favours a scenario where only the fragments that are gravitationally bound can further contract to the limit $m(r)=870 M_{\odot}(r / p c)^{1.33}$.

\subsection{How to form bubble N131?}

Based on the intriguing morphological structure and velocity distribution of N131 presented above, we propose that the progenitor of bubble N131 was a filamentary nebula elongated along the direction of the clumps $\mathrm{AD}$ and $\mathrm{BC}$ (see the sketch in Fig. 10). It is likely that within the middle of the filamentary nebula, a cluster of massive stars ionized, heated, and blew away the surrounding interstellar medium and compressed the preexisting clumps. With the expansion of $\mathrm{H}$ II region at relatively high temperature, pressure, and strong stellar winds lasting more than $10^{6} \mathrm{yr}$, the feedback from the massive stars broke up the pre-existing filamentary nebula and separated it into the two unconnected clumps $\mathrm{AD}$ and $\mathrm{BC}$. With the expansion of evolving $\mathrm{H}$ II region, a ring-like structure is formed. Bodenheimer et al. (1979) have simulated a similar scenario. We provide further evidence to back up this argument.

Clumps $\mathrm{AD}$ and $\mathrm{BC}$ are two unconnected filamentary nebula on the opposite sides of the bubble. Along the direction of clumps C, B, A, and D in Fig. 3, there is a velocity gradient from -14.5 to $-6.5 \mathrm{~km} \mathrm{~s}^{-1}$ (Zhang et al. 2013). This velocity coherence indicates that in the past clumps $\mathrm{AD}$ and $\mathrm{BC}$ were likely connected together. Therefore, the velocity gradient may be partly produced by the bubble expansion.

These broken filamentary morphologies, caused by bubble expansion, are rather common in large-scale surveys, i.e. the 500 pc filamentary gas wisp from Li et al. (2013) and the bubble N39 from Deharveng et al. (2010). In addition, the secondary bubble N131-A (see Sect. 3.7) close to N131 is also embedded 


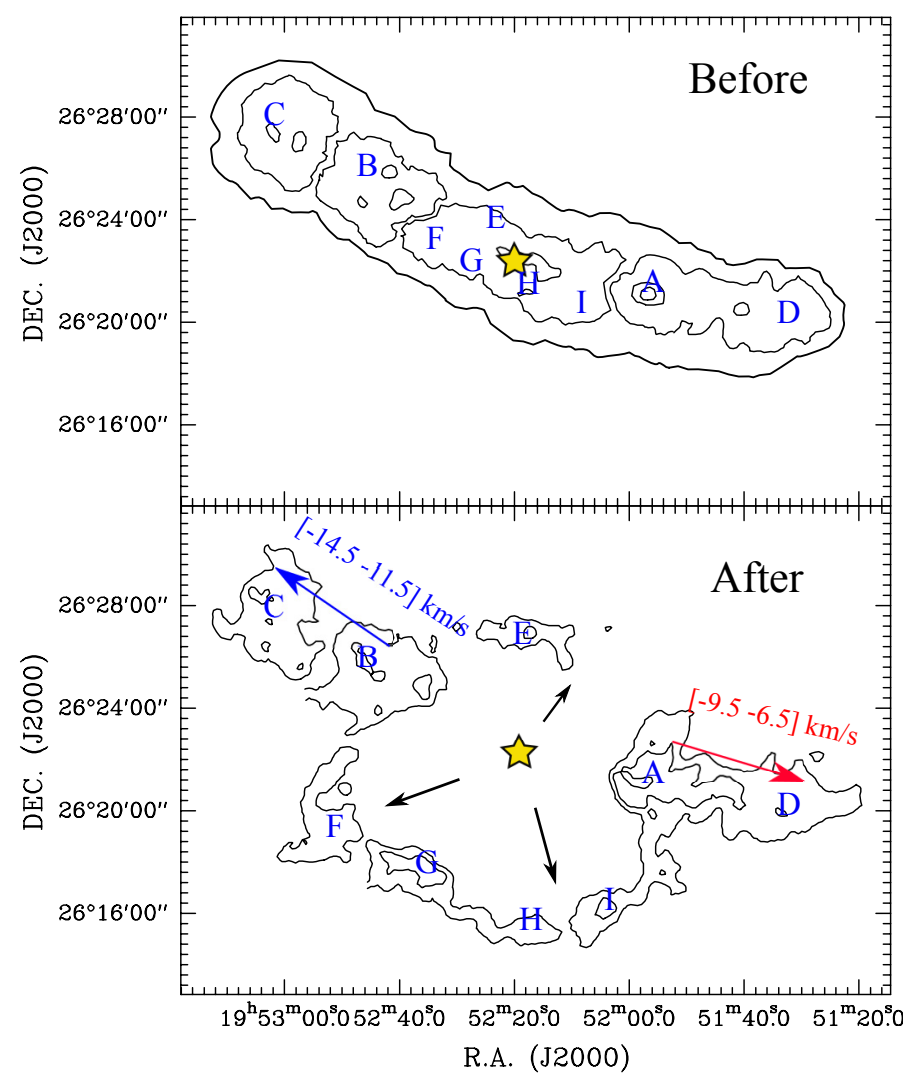

Fig. 10. A schematic illustration depicting our target region before and after the formation of bubble N131. The yellow star indicates a group of ionizing stars that caused the expansion of the shell. In the upper panel, we indicate the proposed pre-existing clumps A to I. In the lower panel, we indicate the observed clumps A to I and the velocity structure.

in the filamentary nebula (or clumps AD); N131-A is expanding outwardly, and compressing the surrounded material. Bubble N131 in its early stage might have had a similar evolutionary process as N131-A. With the expansion of the bubble, the strong stellar winds finally help to form bubble N131.

To test our scenario, we also compared the relative masses of these clumps surrounding bubble N131 with the proposed pre-existing clumps in Fig. 11. These clumps are divided into three parts: clump AD, clump BC, and the ring-like shell (mainly clumps E, F, G, H, and I). The masses of clumps E, F, G, H, and $\mathrm{I}$ in the ring-like shell are mainly from the proposed pre-existing clump $\mathrm{AB}$. Considering that clumps $\mathrm{AD}, \mathrm{BC}$, and $\mathrm{AB}$ have a similar projection area, we assumed that clumps $\mathrm{AD}, \mathrm{BC}$, and $\mathrm{AB}$ had the same mass when it was a connected filamentary nebula in the past. The mass distributions of the three parts are shown in Fig. 11. Clump AD has a very similar mass to clump $\mathrm{BC}$, while the mass of the ring-like shell is a little lower (about $16 \%$ ) than clumps $\mathrm{AD}$ and $\mathrm{BC}$. The $16 \%$ reduction can be explained as some masses from the assumed filamentary clump AB being merged into clumps A and B. Above all, the similarity of the masses of clump AD, clump BC, and the ring-like shell suggests that the bubble might originate from a filamentary nebula if mass conserves.

\section{Summary}

Bubble N131 shows an intriguing morphology and hierarchical velocity distribution, which have been reported in the context of

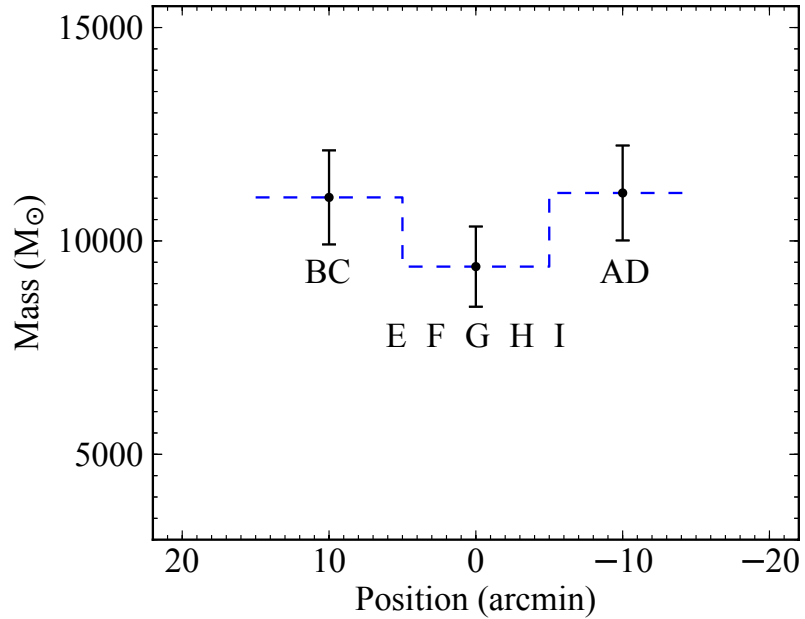

Fig. 11. Mass-position distributions of the clumps A to I in bubble N131. The position axis of the labelled clumps is offset from the bubble centre. Filaments $\mathrm{AD}, \mathrm{BC}$, and the pre-existing clump $\mathrm{AB}$ have a similar projected length of about 10 arcmin. Filament BC; clumps E, F, G, $\mathrm{H}$, I (i.e. the ring-like shell); and filament $\mathrm{AD}$ have a mass of 11000 , 9000 , and $11000 M_{\odot}$, respectively. The error bar is $10 \%$ for each mass value.

previous $\mathrm{CO}$ observations. However, the angular resolution was low, and the bubble formation is still unclear.

In this work, we analysed archival multiwavelength observations including 3.6, 4.5, 5.8, 8.0, 24, 70, 160, 250, 350, $500 \mu \mathrm{m}$, $1.1 \mathrm{~mm}$, and $21 \mathrm{~cm}$ towards bubble N131. These observations revealed the distributions of the warm and cold dust emission. Bubble N131 is a special object owing to its large hole at $24 \mu \mathrm{m}$ and $21 \mathrm{~cm}$ in the direction of the bubble centre. Comparing the 4.5 and $8.0 \mu \mathrm{m}$ with the $24 \mu \mathrm{m}$ and $21 \mathrm{~cm}$, we found a secondary bubble N131-A located at the filamentary clump AD. The derived Lyman-continuum ionizing photon flux within the N131-A is equivalent to an 09.5 star.

We performed new observations of $\mathrm{CO}(2-1), \mathrm{CO}(1-0)$, and ${ }^{13} \mathrm{CO}(1-0)$ using the IRAM $30 \mathrm{~m}$ telescope. The distribution of molecular gas is largely consistent with the dust emission from $5.8 \mu \mathrm{m}$ to $500 \mu \mathrm{m}$. The dust and gas clumps are compact and might have been compressed at the inner edge of the ring-like shell, while they are extended and might be pre-existing at the outer edge. The column density, excitation temperature, and velocity also show a potentially hierarchical distribution from the inner to outer edges of the ring-like shell. We detected the front and back sides of the secondary bubble N131-A in the direction of its centre. Based on the above, we propose a scenario in which bubble N131 was formed by the expansion of H II region and strong stellar winds from a group of massive stars inside the bubble.

Comparing the mass-size relation with high-mass formation threshold $m(r)=870 M_{\odot}(r / \mathrm{pc})^{1.33}$, the bubble is fragmenting into dense fragments which can potentially form low-mass stars. These molecular clumps fragmented into 31 fragments, most of which are located at the two giant filamentary nebula (or the clumps $\mathrm{AD}$ and $\mathrm{BC}$ ). Twelve of the fragments are gravitationally bound and 19 are gravitationally unbound. From the morphology, infrared dust emission, velocity dispersion, and mass distribution, bubble N131 likely originated in a filamentary nebula, within which the strong stellar winds from a group of massive stars broke up a pre-existing filamentary nebula into clumps AD 
and $\mathrm{BC}$, and swept up the surrounded material into a ring-like shell of bubble N131.

Acknowledgements. We wish to thank the anonymous referee for comments and suggestions that improved the clarity of the paper. C.-P. Zhang acknowledges support by the MPG-CAS Joint Doctoral Promotion Program (DPP) and observations by the IRAM staff. This work is partly supported by the National Key Basic Research Program of China (973 Program) 2015CB857100 and National Natural Science Foundation of China 11363004 and 11403042.

\section{References}

Aguirre, J. E., Ginsburg, A. G., Dunham, M. K., et al. 2011, ApJS, 192, 4 Anderson, L. D., Zavagno, A., Deharveng, L., et al. 2012, A\&A, 542, A10 Beaumont, C. N., \& Williams, J. P. 2010, ApJ, 709, 791

Benjamin, R. A., Churchwell, E., Babler, B. L., et al. 2003, PASP, 115, 953 Bodenheimer, P., Tenorio-Tagle, G., \& Yorke, H. W. 1979, ApJ, 233, 85

Cappa, C. E., Rubio, M., Romero, G. A., Duronea, N. U., \& Firpo, V. 2014, A\&A, 562, A6

Carey, S. J., Noriega-Crespo, A., Mizuno, D. R., et al. 2009, PASP, 121, 76

Castor, J., McCray, R., \& Weaver, R. 1975, ApJ, 200, L107

Churchwell, E., Povich, M. S., Allen, D., et al. 2006, ApJ, 649, 759

Churchwell, E., Watson, D. F., Povich, M. S., et al. 2007, ApJ, 670, 428

Churchwell, E., Babler, B. L., Meade, M. R., et al. 2009, PASP, 121, 213

Condon, J. J., Cotton, W. D., Greisen, E. W., et al. 1998, AJ, 115, 1693

Csengeri, T., Bontemps, S., Schneider, N., Motte, F., \& Dib, S. 2011, A\&A, 527, A135

Deharveng, L., Schuller, F., Anderson, L. D., et al. 2010, A\&A, 523, A6

Dyson, J. E., \& Williams, D. A. 1980, Physics of the interstellar medium (Manchester University Press)

Evans, II, N. J. 1999, ARA\&A, 37, 311

Faimali, A., Thompson, M. A., Hindson, L., et al. 2012, MNRAS, 426, 402

Garden, R. P., Hayashi, M., Hasegawa, T., Gatley, I., \& Kaifu, N. 1991, ApJ, 374,540

Ginsburg, A., Glenn, J., Rosolowsky, E., et al. 2013, ApJS, 208, 14

Gómez, L., Wyrowski, F., Pillai, T., Leurini, S., \& Menten, K. M. 2011, A\&A, 529, A161

Goodman, A. A., Alves, J., Beaumont, C. N., et al. 2014, ApJ, 797, 53

Griffin, M. J., Abergel, A., Abreu, A., et al. 2010, A\&A, 518, L3

Hindson, L., Thompson, M. A., Urquhart, J. S., et al. 2013, MNRAS, 435, 2003

Hou, L. G., \& Gao, X. Y. 2014, MNRAS, 438, 426

Jackson, J. M., Finn, S. C., Chambers, E. T., Rathborne, J. M., \& Simon, R. 2010, ApJ, 719, L185
Jones, A., Frey, V., Verstraete, L., Cox, P., \& Demyk, K. 1999, in The Universe as Seen by ISO, eds. P. Cox, \& M. Kessler, ESA SP, 427, 679

Kainulainen, J., Ragan, S. E., Henning, T., \& Stutz, A. 2013, A\&A, 557, A120

Kauffmann, J., \& Pillai, T. 2010, ApJ, 723, L7

Kramer, C., Stutzki, J., Rohrig, R., \& Corneliussen, U. 1998, A\&A, 329, 249

Krumholz, M. R., \& Matzner, C. D. 2009, ApJ, 703, 1352

Lee, H.-T., \& Chen, W. P. 2007, ApJ, 657, 884

Lefloch, B., \& Lazareff, B. 1994, A\&A, 289, 559

Li, G.-X., Wyrowski, F., Menten, K., \& Belloche, A. 2013, A\&A, 559, A34

Martins, F., Pomarès, M., Deharveng, L., Zavagno, A., \& Bouret, J. C. 2010, A\&A, 510, A32

Mezger, P. G., \& Henderson, A. P. 1967, ApJ, 147, 471

Mezger, P. G., Smith, L. F., \& Churchwell, E. 1974, A\&A, 32, 269

Nagai, T., Inutsuka, S.-I., \& Miyama, S. M. 1998, ApJ, 506, 306

Padoan, P., Juvela, M., Goodman, A. A., \& Nordlund, Å. 2001, ApJ, 553, 227

Panagia, N. 1973, AJ, 78, 929

Peng, T.-C., Wyrowski, F., van der Tak, F. F. S., Menten, K. M., \& Walmsley, C. M. 2010, A\&A, 520, A84

Peretto, N., Fuller, G. A., André, P., et al. 2014, A\&A, 561, A83

Poglitsch, A., Waelkens, C., Geis, N., et al. 2010, A\&A, 518, L2

Preibisch, T., Roccatagliata, V., Gaczkowski, B., \& Ratzka, T. 2012, A\&A, 541, A132

Ragan, S. E., Henning, T., Tackenberg, J., et al. 2014, A\&A, 568, A73

Rathborne, J. M., Jackson, J. M., \& Simon, R. 2006, ApJ, 641, 389

Simon, R., Jackson, J. M., Rathborne, J. M., \& Chambers, E. T. 2006, ApJ, 639, 227

Simpson, R. J., Povich, M. S., Kendrew, S., et al. 2012, MNRAS, 424, 2442

Smith, R. J., Glover, S. C. O., Clark, P. C., Klessen, R. S., \& Springel, V. 2014, MNRAS, 441, 1628

Stutzki, J., \& Guesten, R. 1990, ApJ, 356, 513

Wang, K., Zhang, Q., Wu, Y., \& Zhang, H. 2011, ApJ, 735, 64

Wang, K., Zhang, Q., Testi, L., et al. 2014, MNRAS, 439, 3275

Watson, C., Povich, M. S., Churchwell, E. B., et al. 2008, ApJ, 681, 1341

Weaver, R., McCray, R., Castor, J., Shapiro, P., \& Moore, R. 1977, ApJ, 218, 377

Whitworth, A. P., Bhattal, A. S., Chapman, S. J., Disney, M. J., \& Turner, J. A. 1994a, A\&A, 290, 421

Whitworth, A. P., Bhattal, A. S., Chapman, S. J., Disney, M. J., \& Turner, J. A. 1994b, MNRAS, 268, 291

Winnewisser, G., Churchwell, E., \& Walmsley, C. M. 1979, A\&A, 72, 215

Yuan, J.-H., Wu, Y., Li, J. Z., \& Liu, H. 2014, ApJ, 797, 40

Zhang, C. P., \& Wang, J. J. 2012, A\&A, 544, A11

Zhang, C.-P., \& Wang, J.-J. 2013, Res. Astron. Astrophys., 13, 47

Zhang, C.-P., Wang, J.-J., \& Xu, J.-L. 2013, A\&A, 550, A117 